\title{
Performance of Toddlers, Children and Young Adults Using Unilateral or Bilateral Cochlear Implants on a Left versus Right Loudspeaker Identification Task
}

\author{
Karyn L. Galvin Kathryn C. Hughes Jennifer Holland Mansze Mok \\ Audiology, Hearing and Speech Science, The University of Melbourne, Parkville, Vic., Australia
}

\section{Key Words}

Hearing impairment $\cdot$ Cochlear implant • Bilateral cochlear implants $\cdot$ Children $\cdot$ Localisation • Lateralisation

\begin{abstract}
Thirty-six participants with bilateral cochlear implants aged 1-19 years completed a left versus right loudspeaker identification task. The majority performed at chance in the unilateral condition $(n=24)$ and significantly above chance in the bilateral condition $(n=28)$. Cluster analysis identified three groups; one group performed above chance in both conditions and was older at second implant and older at testing, with longer delay between implants. There were no such differences between the group performing at chance in both conditions and the group which scored highly in the bilateral condition only, thus demonstrating a bilateral benefit. Unilateral, but not bilateral, scores were correlated with age at second and at first implant, time between implants, and age at testing. Bilateral benefit was negatively correlated with age at second implant, time between implants and age at testing. Co-linearity made it difficult to isolate the relationships with demographic factors, though age at testing may have had the most influence on unilateral scores. Spatial hearing skills with bilateral implants cannot be predicted for individuals.

Copyright $\odot 2011$ S. Karger AG, Basel
\end{abstract}




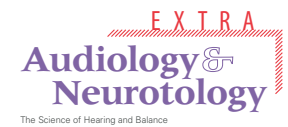

Audiol Neurotol Extra 2011;1:30-44

DOI: 10.1159/000335170

Published online: December 29, 2011

2011 S. Karger AG, Basel

www.karger.com/aue

Galvin et al.: Loudspeaker Identification by Bilaterally Implanted Toddlers to Young Adults

\section{Introduction}

The ability to locate the source of a sound, to localise, is a very valuable skill. Using localisation abilities, the listener is able to create a spatial map of the auditory environment, thus contributing to their connectedness with that environment and increasing their awareness of any changes therein. Localisation is valuable in communication, as it helps the listener to turn in the correct direction when a speaker seeks to attract their attention, and to locate an individual speaker in a group conversation. Localisation also helps the listener to more easily segregate speech and noise arriving from different locations. There are many other ways in which being able to localise a sound increases safety and/or is simply useful; examples include looking in the appropriate direction when in traffic or when a warning signal is heard, being aware if a person is approaching when footsteps are heard, and locating a ringing phone handset.

For listeners with normal acoustic hearing bilaterally, interaural cues are the primary cues to the location of sound sources. For this reason, the localisation ability of children receiving bilateral cochlear implants (CIs) has been assessed as a measure of the additional benefit provided by two CIs in comparison to one. Researchers have sometimes also attempted to relate performance to demographic characteristics, such as the age at implantation. Determining the impact of demographic characteristics on outcomes is important for increasing the evidence base for informing the pre-operative counselling provided to families considering bilateral implantation. As would be expected, assessment methodologies have varied across studies examining the localisation abilities of children using bilateral implants. One key reason for this has been the testing capabilities of different age groups.

Assessments of localisation performance for adults using bilateral CIs have generally required the participant to select the presenting loudspeaker from a frontal multi-loudspeaker array positioned in the horizontal plane [see, for example, Laske, 2009; van Hoesel and Tyler, 2003; Verschuur et al., 2005]. In contrast, there are only four paediatric reports which have used this type of task [Galvin et al., 2007a, b; Grieco-Calub and Litovsky, 2010; Van Deun et al., 2010]. These studies used $120-180^{\circ}$ arrays of 7-15 loudspeakers, and involved only children aged around 4-15 years. In the first study, unilateral and bilateral performance was generally poor, with a group $(n=8)$ root mean square $(R M S)$ error around $69^{\circ}$, and little evidence of bilateral benefit [Galvin et al., 2007a]. The older age at second implant (around 6-14 years) and minimal bilateral experience may have contributed to the poor outcome, though a second report of 6 participants re-assessed at 12 months also indicated no bilateral benefit [Galvin et al., 2007b]. The two more recent studies involved larger participant groups (21 and 30, respectively), and had more positive and relatively consistent outcomes [GriecoCalub and Litovsky, 2010; Van Deun et al., 2010]. The group's bilateral RMS error was around $38^{\circ}$, though individual scores ranged from $13^{\circ}$ to $63^{\circ}$. Where unilateral performance was assessed, around half of the participants showed a bilateral benefit of $9-23^{\circ}$ [Grieco-Calub and Litovsky, 2010]. The inclusion of participants who received a second implant before 5 years of age, and/or who had more bilateral experience, as well as pre-test training [only in Van Deun et al., 2010] and feedback during testing, may have contributed to the superior result in these studies as compared with Galvin et al. [2007a, b].

As noted above, the studies reporting results with a multi-loudspeaker array included mostly children aged 5 years and over. This is not surprising, given that the task is difficult to understand, the procedure is time-consuming to complete, and younger children may be unable to provide a response that can be interpreted. It can be difficult to maintain the engagement of implanted children on this type of task when their localisation skills are poor, and this difficulty is likely to increase with decreasing age. The implication of this is that data are less likely to be collected from children with poorer spatial hearing skills. 
Studies which have used a simpler and likely shorter protocol employing just three loudspeakers have been able to include slightly younger children. Lovett et al. [2010] used loudspeakers positioned at $0^{\circ}$ and $\pm 60^{\circ}$ to evaluate children aged $4-16$ years. The median score was significantly higher for the bilateral $(42.5 \% ; \mathrm{n}=20)$ versus unilateral $(71.7 \%)$ groups, with some overlap of individual scores within the groups. Steffens et al. [2008] used loudspeakers positioned at $0^{\circ}$ and $\pm 90^{\circ}$ to evaluate 19 children aged 3.9-10.6 years. Bilateral scores were above chance for $95 \%$ of participants. As unilateral scores were above chance for $79 \%$, a bilateral benefit was gained by only one-third of participants.

Even with three loudspeakers, testing has been limited to children at least 3.9 years of age and older. Very young children need a test protocol which does not require a verbal explanation. When just two loudspeakers are used, the test protocol can be based on Visual Reinforcement Audiometry (VRA), the standard hearing testing approach for younger children. It is important to note that, with this simplification of the testing approach, the absolute ability to identify the location of a sound source is no longer being assessed. Rather, the listener is making a relative decision in a two-alternative, forced-choice task. Although this is generally considered to be an assessment of spatial hearing abilities, the relationship between performance on this task and localisation in everyday life is not at all clear. One pair of loudspeakers may be at a fixed angle of separation, or multiple pairs may be positioned at a variety of angles of separation. This latter type of setup enables calculation of the minimum audible angle (MAA), the smallest angle of separation at which the listener can make the left versus right discrimination.

Litovsky and colleagues measured the MAA using a within-subject design for older children aged 3-16 years [Litovsky et al., 2006] and a between-subject design for younger children aged 2-3 years [Grieco-Calub et al., 2008]. Of the 13 older children, 4 found the task very difficult; of these, 1 could not lateralise even in the bilateral condition and 3 (aged 4-6 years) could not lateralise in the unilateral condition. For the remaining 9 participants, the loudspeaker location at which the bilateral MAA was measured ranged from $\pm 5^{\circ}$ to $40^{\circ}$, and the bilateral MAA was $10-50^{\circ}$ smaller than the unilateral MAA. A significant bilateral benefit (around $33^{\circ}$ ) was found for the group of 5 participants with less than 13 months bilateral experience, but not the 4 participants with more than 13 months experience. In the study involving participants aged 2-3 years, the MAA could not be measured for any of the 8 unilaterally implanted participants, and could only be measured for 5 bilaterally implanted participants with a fixed intensity stimulus (loudspeaker location $\pm 7.5-40^{\circ}$ ) and for 4 with stimulus jitter (loudspeaker location $\pm 7.5-52.5^{\circ}$ ). Sparreboom et al. [2011] employed a MAA task with 29 sequentially implanted children aged $4.8-10.5$ years. The mean loudspeaker position for the unilateral MAA was $\pm 78^{\circ}$, with only $41 \%$ of participants able to lateralise above chance. The mean bilateral MAA was significantly smaller, with the loudspeakers at $\pm 42^{\circ}$ (range: $\pm 15-90^{\circ}$ ) and $83 \%$ of participants able to lateralise above chance.

To summarise these MAA studies, group performance was superior in the bilateral condition across all studies, with more participants able to lateralise, and the MAA measured for loudspeakers positioned at smaller angles. One or two participants even performed within the range shown by children with normal hearing; however, in each study there were poor performers who were unable to lateralise even in the bilateral condition. In addition, where individual unilateral and bilateral data was presented, around $65-75 \%$ of participants did not appear to demonstrate a bilateral benefit. Performance in the unilateral condition was more likely to be poor for children under 6 years of age.

The simplest assessment protocols use just one pair of loudspeakers to evaluate lateralisation ability. Beijen et al. [2007] evaluated 5 participants aged 2.5-6.6 years with loudspeakers positioned at $\pm 30^{\circ}$ and $\pm 90^{\circ}$. Unilateral scores for the bilateral participants and a unilateral group were at chance level, whilst bilateral scores were well above chance. This bilat- 
eral group was unusual in that none had a congenital onset, the period of deafness was very short, and 4 participants were simultaneously implanted. The task was also made easier by the use of a fixed-intensity stimulus and a presentation period of up to $5 \mathrm{~s}$. Galvin et al. [2008] used loudspeakers positioned at $\pm 90^{\circ}$ to evaluate 9 children aged $1.8-6$ years, most of whom had just 6 months bilateral experience. All participants performed at chance level in the unilateral condition, whilst 7 of the 9 performed above chance in the bilateral condition. Lovett et al. [2010] evaluated children aged 1.5-16 years. Median scores were significantly higher for the bilateral $(\mathrm{n}=30)$ versus unilateral groups for loudspeakers positioned at $\pm 60^{\circ}(100$ vs. $65 \%$ ) and $\pm 30^{\circ}$ (85 vs. $55 \%$ ), although there was some overlap of individual scores across the two groups. Sparreboom et al. [2011] evaluated 27 children aged 3.8-9.5 years. Pre-operatively, none of 8 participants assessed could lateralise, whilst 12 months after the second implant, $63 \%$ performed above chance on loudspeakers at $\pm 15^{\circ}(11 \%)$, at $\pm 30^{\circ}(15 \%)$, or $\pm 90^{\circ}(37 \%)$. In summary, in these studies employing a pair of loudspeakers, performance was at chance level in the unilateral condition for the majority or all participants. The majority of participants performed above chance in the bilateral condition, though significant inter-subject variation was evident, with some participants able to lateralise at small angles of separation, whilst $25-37 \%$ of participants in two studies performed only at chance level. The participants were mainly limited to children under the age of 10 years, and no studies attempted to relate performance on this task to demographic characteristics.

Given the variability reported across individuals in all of the studies reviewed above, there is a clear need to identify the demographic characteristics which may influence performance. Table 1 summarises the outcomes of the studies which have evaluated the relationship between performance and demographic characteristics of the participants. No factors were identified as likely to have had a consistent impact on unilateral performance; where a relationship was identified as being significant, the authors suggested that it was due to the influence of a particular group of participants [Steffens et al., 2008] or the related variable of age at testing [Sparreboom et al., 2011]. There was not one factor which all studies identified as being related to bilateral performance; in each case where a relationship was identified, other studies found no relationship. The factors for which a significant relationship with bilateral performance was found included early auditory stimulation, as represented by age at first implant (CI-1) or duration of pre-implant hearing aid use [Van Deun et al., 2010], bilateral experience [Steffens et al., 2008], and age at second implant (CI-2) [Grieco-Calub and Litovsky, 2010; Steffens et al., 2008; Van Deun et al., 2010]. Both Van Deun et al. [2010] and Grieco-Calub and Litovsky [2010] raised the issue of the relationships between the factors of age at CI-1, age at CI-2, and age at testing, and how the high correlations amongst these factors made it difficult to isolate the influence of any one factor. None of the studies reported on the relationship between age at testing and bilateral performance, and none considered the relationships between any demographic characteristics and bilateral benefit. In addition, those studies which considered the relationship between demographic characteristics and performance only included children aged 3.9-15 years.

The studies in the literature evaluating spatial hearing abilities of children with bilateral implants have established that performance is typically better with two implants compared with one; however, outcomes vary greatly across individuals, and the relationship between demographic characteristics and performance, particularly bilateral benefit, is not clear. Clarifying these relationships has been made more difficult by the limited age range (generally over 5 years) of participants in the studies. The main aim of the present study was to evaluate the ability to discriminate stimuli presented from the left or right, using one or two CIs, for participants of a wide age range from toddlers through to young adults. Further aims were to determine the degree of bilateral benefit gained, and to consider the relationship between performance and demographic characteristics. 


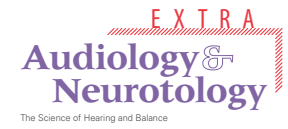

\begin{tabular}{l}
\hline \begin{tabular}{l} 
Audiol Neurotol Extra 2011;1:30-44 \\
\hline DOI: 10.1159/000335170
\end{tabular} \\
\begin{tabular}{ll} 
Published online: December 29, 2011 & $\begin{array}{l}\text { (2011 S. Karger AG, Basel } \\
\text { www.karger.com/aue }\end{array}$ \\
\hline Galvin et al.: Loudspeaker Identification by Bilaterally Implanted Toddlers to Young Adults
\end{tabular}
\end{tabular}

Table 1. Localisation and lateralisation studies which have evaluated the relationship between performance and demographic characteristics of the participants (relationships with bilateral benefit were not evaluated)

\begin{tabular}{|c|c|c|}
\hline \multirow[t]{2}{*}{ Demographic characteristic } & \multicolumn{2}{|c|}{ Studies evaluating the relationship between demographics and performance } \\
\hline & unilateral performance & bilateral performance \\
\hline Age at onset & Steffens $[2008](3)[-v e]^{1}$ & $\begin{array}{l}\text { Steffens [2008] } \\
\text { Van Deun [2010] }\end{array}$ \\
\hline Aetiology & Steffens $[2008](3)[-v e]^{1}$ & Steffens [2008] \\
\hline History of acoustic hearing ${ }^{2}$ & Grieco-Calub [2010] & Grieco-Calub [2010] \\
\hline $\begin{array}{l}\text { Use of pre-implant hearing aid } \\
\text { for more than } 18 \text { months }\end{array}$ & & Van Deun [2010] (9) [+ve] \\
\hline Age at CI-1 & $\begin{array}{l}\text { Steffens [2008] } \\
\text { Sparreboom [2011] } \\
\text { Grieco-Calub [2010] }\end{array}$ & $\begin{array}{l}\text { Steffens }[2008] \\
\text { Sparreboom }[2011] \\
\text { Van Deun }[2010](\mathbf{9})\left[+\mathbf{~ e ]}\left(<2 \text { years }^{3}\right)\right.\end{array}$ \\
\hline Time between CIs & $\begin{array}{l}\text { Steffens [2008] } \\
\text { Grieco-Calub [2010] }\end{array}$ & $\begin{array}{l}\text { Steffens [2008] } \\
\text { Grieco-Calub [2010] } \\
\text { Van Deun [2010] }\end{array}$ \\
\hline Age at CI-2 & $\begin{array}{l}\text { Steffens }[2008] \\
\text { Sparreboom }[2011](2, \text { MAA })[+\mathbf{v e}]^{4} \\
\text { Grieco-Calub }[2010]\end{array}$ & $\begin{array}{l}\text { Steffens }[2008](3)[-v e] \\
\text { Sparreboom }[2011] \\
\text { Grieco-Calub }[2010](7 \text { or 15) }[- \text { ve }] \\
\text { Van Deun }[2010](9)[- \text { ve }](<4 \text { years })^{6}\end{array}$ \\
\hline Bilateral experience & $\begin{array}{l}\text { Steffens [2008] } \\
\text { Grieco-Calub [2010] }\end{array}$ & $\begin{array}{l}\text { Steffens [2008] (3) [+ve] } \\
\text { Grieco-Calub [2010] } \\
\text { Van Deun [2010] }\end{array}$ \\
\hline Age at testing & Grieco-Calub [2010] & \\
\hline $\begin{array}{l}\text { Studies are identified by the } \\
\text { was reported; for these studies b } \\
\text { indicate the direction of the corr } \\
{ }^{1} \text { Influenced by relatively poor } \\
{ }^{2} \text { Defined as some level of usa } \\
{ }^{3} \text { Performance of children rec } \\
\text { planted after } 2 \text { years of age. } \\
{ }^{4} \text { The authors suggested older } \\
\text { nent of the MAA task. } \\
{ }^{5} \text { Performance of children rec } \\
\text { planted after } 4 \text { years of age. } \\
{ }^{6} \text { The authors suggested that } \mathrm{t} \\
\text { of age at CI-1 and age at testing. }\end{array}$ & $\begin{array}{l}\text { first author and year of publication. B } \\
\text { brackets indicate the number of loudsp } \\
\text { relation. } \\
\text { r performance of } 4 \text { participants who ha } \\
\text { able hearing (with or without hearing a } \\
\text { ceiving CI- } 1 \text { before } 2 \text { years of age demor } \\
\text { r children demonstrated superior unila } \\
\text { ceiving CI- } 2 \text { before } 4 \text { years of age demor } \\
\text { the effect of age at CI- } 2 \text { was underestim }\end{array}$ & $\begin{array}{l}\text { d type indicates a significant correlation } \\
\text { kers used in testing and square brackets } \\
\text { a non-congenital onset due to meningitis. } \\
\text { is) before deafness and implantation. } \\
\text { trated superior performance to those im- } \\
\text { ral performance due to cognitive compo- } \\
\text { trated superior performance to those im- } \\
\text { ed due to high correlation with covariates }\end{array}$ \\
\hline
\end{tabular}

\section{Method}

\section{Participants}

Results are reported here for 36 participants. Results for 5 participants were also included in an earlier report [Galvin et al., 2008]. Additional participants could not be conditioned $(n=1)$ or could not cooperate for a sufficient length of time to complete an assessment $(n=2)$.

All participants had a profound bilateral hearing loss prior to 2 years of age. For 32 participants the onset was congenital, the aetiology being unknown (17; 3 of these children were 


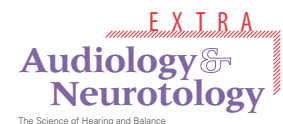

\begin{tabular}{l|l}
\hline Audiol Neurotol Extra 2011;1:30-44 \\
\hline $\begin{array}{l}\text { DOI: 10.1159/000335170 } \\
\text { Published online: December 29, } 2011\end{array}$ & $\begin{array}{l}\text { @ } 2011 \text { S. Karger AG, Basel } \\
\text { www.karger.com/aue }\end{array}$ \\
\hline
\end{tabular}

Galvin et al.: Loudspeaker Identification by Bilaterally Implanted Toddlers to Young Adults

Table 2. Demographic characteristics of the 36 participants

\begin{tabular}{ll}
\hline Mean age \pm SD, years (range) & \\
At CI-1 & $2.1 \pm 2.1(0.6-11.3)$ \\
At CI-2 & $5.8 \pm 5.1(0.8-19.8)$ \\
At testing & $6.9 \pm 5.3(1.3-20.8)$ \\
Mean time between implants \pm SD, years (range) & $3.7 \pm 3.9(0-16.7)^{\mathrm{a}}$ \\
Mean bilateral experience $\pm \mathrm{SD}$, years (range) & $1.0 \pm 0.6(0.5-2.1)$ \\
\hline
\end{tabular}

${ }^{a}$ Four participants were simultaneously implanted.

diagnosed with auditory neuropathy), Connexin 26 (5), other genetic causes (4; for 1 of these children the hearing loss was initially severe), KIDD syndrome (2), Cytomegalovirus (2), or wide vestibular aqueduct syndrome (2). For an additional 2 participants the onset was possibly congenital, although the diagnosis was not made until after 14 months and the aetiology was unknown. The final 2 participants had contracted bacterial meningitis at 9 and 21 months, respectively. Table 2 presents the age at implant, age at testing, time between implants, and bilateral experience of the group. Only 6 participants consistently wore a contralateral hearing aid prior to CI-2; the unaided, three-frequency average in the second implanted ear for these 6 ranged from 98 to $118 \mathrm{dBA}$ (mean $=105 \mathrm{~dB} ; \mathrm{SD}=7.1$ ). All participants were implanted bilaterally with nucleus CIs. Three older participants had a CI22 implant in their first implanted ear and a CI24 implant in their second implanted ear. The remaining 33 participants had CI24 implants bilaterally.

\section{Assessment}

The testing schedule included assessment points at 6,12 and 24 months after switch-on. Once an individual achieved a score of $>90 \%$ in the bilateral condition, the test was not administered at the next assessment point. The actual assessments completed by an individual depended on the availability of the family and, for younger children, the child's cooperation in completing the testing. Reported here is one assessment result for each individual, this being the latest result obtained for that individual.

All testing was conducted in a low-reverberation sound-proof booth. A Tannoy Reveal loudspeaker was positioned at ear level at $90^{\circ}$ to the right and to the left of the subject at a distance of $115 \mathrm{~cm}$. The auditory stimulus was a series of 4 pink-noise bursts, each of $170 \mathrm{~ms}$ duration with $10 \mathrm{~ms}$ rise and fall times, with an inter-burst duration of $50 \mathrm{~ms}$. The presentation level was $62 \mathrm{dBA}$, with a jitter of $8 \mathrm{~dB}$ to limit the use of monaural loudness cues. All participants were assessed using both CIs (bilateral condition) and one CI (unilateral condition). For the unilateral condition, sequentially implanted participants were assessed using CI-1. Three of the 4 simultaneously implanted participants were assessed using each CI alone, with the mean result reported here. The fourth simultaneously implanted participant only managed to complete testing using the right CI alone. Thirty-five participants completed 2 test blocks involving each of the 2 device conditions, with the order of conditions balanced across test blocks; 1 participant completed only 1 test block due to fatigue. For each new device condition, the child was engaged in a minimum of 5 min conversation or 'conversational play' prior to testing. As detailed below, other aspects of the assessment procedure differed depending upon the child's developmental stage and concentration span.

Older (Generally over 5 Years Old) Participants $(n=19)$

The participant sat on an office chair facing $0^{\circ}$. Prior to testing, the listener was exposed to the stimulus; with the tester identifying the presenting loudspeaker, one stimulus was pre- 
sented from each loudspeaker. A second presentation was then made from each loudspeaker. This process was repeated with each change of device condition. Testing then commenced, with 20 test presentations in each device condition in each of the 2 test blocks, for a total of 40 presentations per condition (2 participants managed only a total of 24 presentations per condition across test blocks). Participants identified the presenting loudspeaker verbally or by pointing.

\section{Younger Participants}

The participant sat on the parent's lap or on a small chair facing an assistant tester sitting at $0^{\circ}$. The assistant tester maintained the child's position and cooperation by verbal instruction and social reinforcement. Testing for these participants was based on the standard approaches to paediatric hearing testing: VRA for the youngest children $(\mathrm{n}=13$; generally those under 4 years) and play audiometry for children $(n=4)$ who were more capable and/ or likely to lose interest in the VRA task.

VRA and Play Audiometry

In both VRA and play audiometry, children are conditioned to make a motor response to a stimulus which the tester is confident is highly salient to the child; this would typically be a relatively high intensity auditory stimulus or a tactile stimulus. Conditioning in the present study required a stimulus with a highly salient directional component, with a flashing light serving this purpose. A coloured flashing light $7 \mathrm{~cm}$ in diameter was positioned below the loudspeakers on the right (blue light) and left (red light). Dim room lighting ensured easy localisation of the light source. At the start of each test session, the child was conditioned to the flashing light paired with the auditory stimulus (light + sound). Conditioning was usually achieved with just 2-3 presentations. Following conditioning, testing commenced. Twelve sound-alone presentations were made in each device condition in each of the 2 test blocks, for a total of 24 presentations per device condition. Due to fatigue, 4 participants managed only a total of 9-21 sound-alone presentations across the 2 test blocks. During testing, blocks of 3 sound-alone presentations were alternated with 3 sound + light presentations, beginning with the easier sound + light condition. This approach was based on pilot work which had indicated that providing the easier sound + light presentations was necessary for subject motivation, and to confirm during testing that the subject remained conditioned and willing to respond; this was particularly important in the unilateral CI condition in which nearly all young children were unable to lateralise. It is important to note that these light + sound presentations were not included in any of the data analyses presented below.

For those assessed using a VRA-style approach, the required response was a head turn to one side to view the reinforcer on the right or left. If the child turned the head in more than one direction, the direction of the initial turn was accepted as the response. Reinforcement was provided for such a head turn irrespective of whether it was to the correct side. Such reinforcement was required to maintain the child's conditioning, particularly in the unilateral condition. The reinforcer was a toy in a smoked-Plexiglas-fronted box which was only visible when illuminated by the tester. There were 4 toys to provide sufficient variety to maintain the child's interest. Part-way through the study the toys were replaced by a software-controlled reward system which displayed simple moving gif images on monitors.

For those assessed using a play-audiometry-style approach, the required response was a motor response such as inserting a peg in a board or adding a sticker to a picture. As is the case in standard play-audiometry, the response tasks were varied by the assistant tester (an experienced clinician) to suit the capabilities and maintain the interest of individual children. Irrespective of the particular task, the child was required to make a clear indication of direction in their response by, for example, pointing at the relevant loudspeaker after placing 


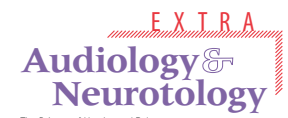

Audiol Neurotol Extra 2011;1:30-44

DOI: $10.1159 / 000335170$

Published online: December 29, 2011

Galvin et al.: Loudspeaker Identification by Bilaterally Implanted Toddlers to Young Adults

the sticker on a picture. Some children required further reward to maintain their cooperation with the task; these children were shown a simple gif image on a monitor positioned above the loudspeaker which they had identified as presenting the sound.

\section{Results}

As noted in the Method section, sound + light presentations were used with young children for the purposes of conditioning, and to maintain and demonstrate the child's continued cooperation with the task during testing. Eighteen participants aged less than 5 years (and 1 child aged 5 years 11 months who required extra encouragement to cooperate with testing) were presented with sound + light stimuli. Sixteen of these participants scored $100 \%$ in the sound + light condition in both device conditions, and only 1 participant scored less than $95 \%$ (that score being $88 \%$ in the unilateral condition). As the purpose of the sound + light presentations was only to promote and demonstrate the child's continued cooperation with the testing, the analysis reported below and the discussion include only results obtained in the sound-alone condition.

Figure 1 presents the percentage of correct responses on the left versus right loudspeaker identification task in the unilateral condition, with participants ordered by age at CI- 2 . Also shown is any gain in the percentage of correct identifications in the bilateral over the unilateral condition (i.e. the bilateral benefit). For the unilateral condition, binomial analysis indicated that the majority of participants $(n=24)$ scored at chance level. For the 12 participants scoring significantly above chance level in the unilateral condition $(\mathrm{p}<0.05$; indicated by the asterisk in fig. 1$)$, the mean score was $84.7 \%(\mathrm{SD}=10.7)$. For the bilateral condition, binomial analysis indicated that the majority of participants $(n=28)$ scored significantly above chance level ( $\mathrm{p}<0.05$; indicated by the cross-hatch in fig. 1 ), with a mean score of $94.1 \%$ $(\mathrm{SD}=9.3)$ for this group. Nineteen of these 28 participants also demonstrated a significant bilateral benefit $(Z \geq-2.19, \mathrm{p} \leq 0.028$; indicated by the caret in fig. 1). Of the 9 who showed no significant bilateral benefit, the potential to demonstrate a significant benefit could possibly have been limited by ceiling effects for 5 participants; these participants scored above $87.5 \%$ in the unilateral condition and above $92.5 \%$ in the bilateral condition.

Given the variation across individuals, an agglomerative hierarchical cluster analysis was conducted on the unilateral and bilateral scores of the 36 individuals to identify any patterns of performance. A cluster analysis is an exploratory technique which organizes individuals into clusters which have high within-group homogeneity and high between-group heterogeneity. The analysis begins with each individual as his/her own cluster and then iteratively combines the most similar clusters; a stopping point for this process is selected based on the similarity within the clusters and the distance between the clusters which are formed. The analysis was performed using Minitab 15 statistical software employing the complete linkage method and the Euclidean distance measure. The analysis identified 3 distinct clusters within the unilateral and bilateral data: Group A $(n=8)$, Group B $(n=17)$, and Group C $(\mathrm{n}=11)$. As was the intended outcome of the cluster analysis, the groups identified by the analysis differed in terms of their performance in the unilateral and/or bilateral conditions (fig. 2). An analysis of variance and post-hoc testing indicated that the performance of Groups A and B was not significantly different $(p=0.97)$ in the unilateral condition, with the performance of Group C being significantly higher than each of the other groups ( $\mathrm{p}<$ 0.001). Post-hoc testing also indicated that the performance of Groups B and C was not significantly different $(\mathrm{p}=0.37)$ in the bilateral condition, with the performance of Group A being significantly poorer than each of the other groups $(\mathrm{p}<0.001)$. The demographic characteristics of these 3 groups, which were formed based on the analysis of the individuals' 


\section{Audiology \\ Neurotology}

\begin{tabular}{l|l}
\hline Audiol Neurotol Extra 2011;1:30-44 \\
\hline DOI: $10.1159 / 000335170$ & $\begin{array}{l}\text { @ } 2011 \text { S. Karger AG, Basel } \\
\text { www.karger.com/aue }\end{array}$ \\
Published online: December 29, 2011 &
\end{tabular}

Galvin et al.: Loudspeaker Identification by Bilaterally Implanted Toddlers to Young Adults

Fig. 1. The percentage of correct responses on the left versus right loudspeaker identification task in the unilateral condition, and any gain in the percentage of correct identifications in the bilateral over the unilateral condition (i.e. the bilateral benefit) for the 36 participants. Participants are ordered by age at CI-2. The black dashed line is the chance level; the score at which performance is significantly above chance level varies depending on the number of stimulus presentations for each individual.

Fig. 2. Percentage of correct responses on the left versus right loudspeaker identification task for Groups A, B and C in the unilateral and bilateral cochlear implant conditions (a and $\mathbf{b}$, respectively). The groups were identified as distinct groups within the performance data by a cluster analysis (conducted by Minitab 15 statistical software and employing the complete linkage method and the Euclidean distance measure). Shown are the median (solid line), interquartile range (box), minimum and maximum (whiskers). Also shown is the bilateral benefit for the three groups (score in bilateral condition minus score in unilateral condition; $\mathbf{c}$.

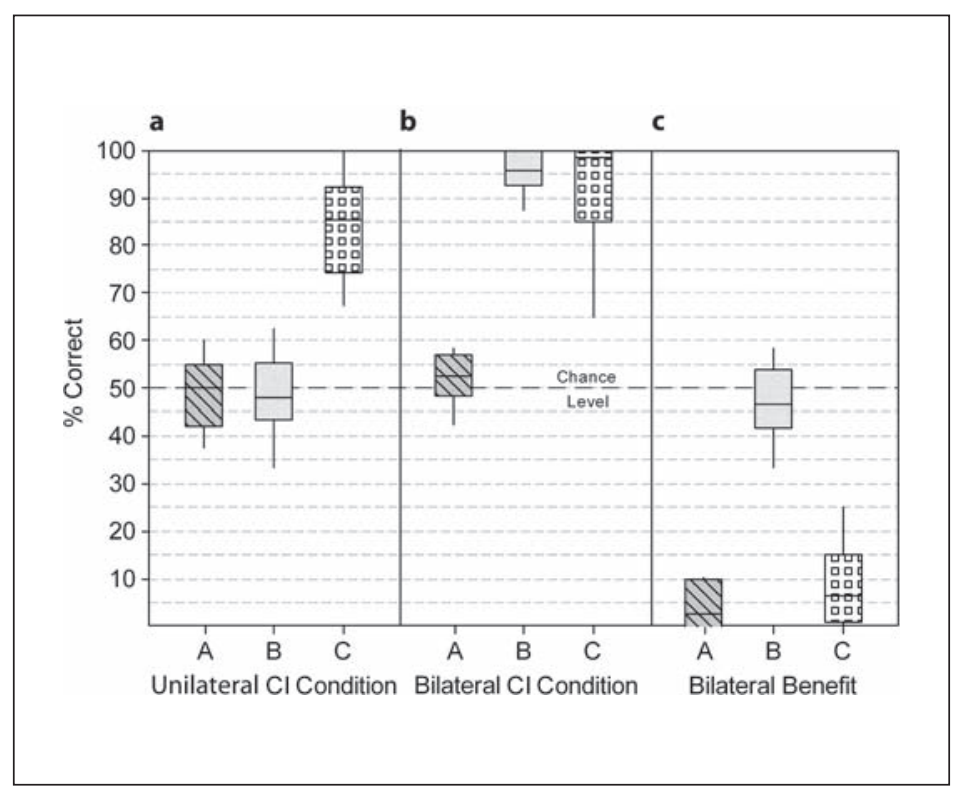

Table 3. Characteristics of the 3 participant groups identified by the cluster analysis conducted on the performance data obtained from all 36 participants in the unilateral and bilateral CI conditions

\begin{tabular}{lllc}
\hline & Group A $(\mathrm{n}=8)$ & Group B $(\mathrm{n}=17)$ & Group C $(\mathrm{n}=11)$ \\
\hline Mean age \pm SD, years & & & \\
$\quad$ At CI-1 & $1.6 \pm 1.0$ & $1.7 \pm 1.4$ & $3.2 \pm 3.0$ \\
At CI-2 & $3.8 \pm 1.9^{\mathrm{C}}$ & $3.2 \pm 2.7^{\mathrm{C}}$ & $11.2 \pm 5.6^{\mathrm{A}, \mathrm{B}}$ \\
At testing & $4.9 \pm 2.3^{\mathrm{C}}$ & $4.0 \pm 2.8^{\mathrm{C}}$ & $12.4 \pm 5.5^{\mathrm{A}, \mathrm{B}}$ \\
Mean time between implants \pm SD, years & $2.1 \pm 1.3^{\mathrm{C}}$ & $1.6 \pm 1.8^{\mathrm{C}}$ & $8.0 \pm 4.1^{\mathrm{A}, \mathrm{B}}$ \\
Mean bilateral experience \pm SD, years & $1.1 \pm 0.5$ & $0.8 \pm 0.5$ & $1.2 \pm 0.6$ \\
Mean bilateral benefit \pm SD, \% & $2.9 \pm 6.3$ & $45.3 \pm 8.8$ & $5.2 \pm 9.4$ \\
\hline
\end{tabular}

A Significantly different to Group A (p $<0.05) .{ }^{\text {B }}$ Significantly different to Group B $(\mathrm{p}<0.05) .{ }^{\text {C Sig- }}$ nificantly different to Group C ( $\mathrm{p}<0.05)$. 
performance data, were then examined (refer to table 3). An analysis of variance and posthoc testing indicated that Groups A and B were not significantly different in terms of any demographic characteristics examined, whilst Group C was significantly older than Groups $\mathrm{A}$ and $\mathrm{B}$ at the time of $\mathrm{CI}-2$, and at testing, and had a longer time between implants $(\mathrm{p}<$ 0.001 ). Note that time between implants may also be considered as length of experience using CI-1 alone. There was no significant difference between the groups for age at CI-1 or bilateral experience $(\mathrm{p} \geq 0.14)$. Bilateral experience was based on the time since CI-2 was switched on; however, it is worth noting that the degree of actual bilateral experience is likely to vary across participants depending on their consistency of use of their two implants together.

Further consideration was then given to the relationship between performance and the demographic characteristics of age at implant, age at testing, and time between implants. Pearson's correlation coefficients indicated a high degree of co-linearity amongst these factors. For example, age at CI-2 was strongly correlated with age at CI-1, age at testing, and time between implants ( $\mathrm{r} \geq 0.74 ; \mathrm{p}<0.001$ ). This was not surprising, given that all sequentially implanted participants were older at the time of CI- 2 than at the time of CI-1, all participants were older at testing than at the time of CI-2, and $92 \%$ of participants were first implanted under 4 years of age, so that those who were older at CI-2 necessarily had a longer time between implants. Similarly, time between implants was strongly correlated with age at testing $(\mathrm{r}=0.93 ; \mathrm{p}<0.001)$ and was also correlated with age at CI-1 $(\mathrm{r}=0.45 ; \mathrm{p}=0.006)$. This high degree of co-linearity amongst factors makes it difficult to validly consider the impact of individual factors, such as may be achieved through a step-wise regression analysis. Thus, whilst acknowledging the co-linearity amongst factors, just Pearson's correlation coefficients were calculated for the relationship between each factor and the performance data for all 36 participants.

The score in the unilateral condition was significantly and strongly associated with age at CI-2, time between implants, and age at testing ( $\mathrm{r} \geq 0.72$; $\mathrm{p} \leq 0.001$ ), and modestly associated with age at CI-1 $(\mathrm{r}=0.46 \mathrm{p}=0.005)$. To remove the impact of participants with potential ceiling effects, the participants $(n=2)$ scoring greater than $95 \%$ in the unilateral condition were removed from the analysis; all correlations remained significant $(r \geq 0.5 ; \mathrm{p} \leq$ 0.003 ). The score in the bilateral condition was not significantly correlated with any of these factors ( $r \leq 0.19 ; \mathrm{p} \geq 0.257$ ). To remove the impact of participants with potential ceiling effects, the participants $(n=13)$ scoring greater than $95 \%$ in the bilateral condition were removed from the analysis; all correlations remained non-significant $(\mathrm{r} \leq-0.13$; $\mathrm{p} \geq 0.53$ ). Bilateral benefit was significantly negatively, though modestly, associated with time between implants, age at CI-2, and age at testing $(\mathrm{r} \geq-0.44 \mathrm{p} \leq 0.007)$, but not with age at CI-1 $(\mathrm{r}=$ $-0.24 ; \mathrm{p}=0.156)$. To remove the impact of participants with potential ceiling effects, the participants $(\mathrm{n}=8)$ whose bilateral benefit may have been limited by their high score in the unilateral $(\geq 80 \%)$ and/or bilateral condition $(\geq 92.5 \%)$ were removed from the analysis; all significant correlations remained significant $(\mathrm{r} \geq-0.38, \mathrm{p} \leq 0.047)$. As an example of the relationship between the performance data and demographic characteristics, figure 3 presents scatterplots of scores in the unilateral and bilateral conditions and the bilateral benefit as a function of age at CI-2. The pattern of the association is similar for the other demographic characteristics.

\section{Observations from Test Sessions}

An interesting observation from test sessions was that participants who scored at chance level in the unilateral condition and above chance in the bilateral condition demonstrated clear behavioural differences in the 2 device conditions. In the bilateral condition the participants quickly and confidently turned to, or indicated, the (usually) correct loudspeaker. 


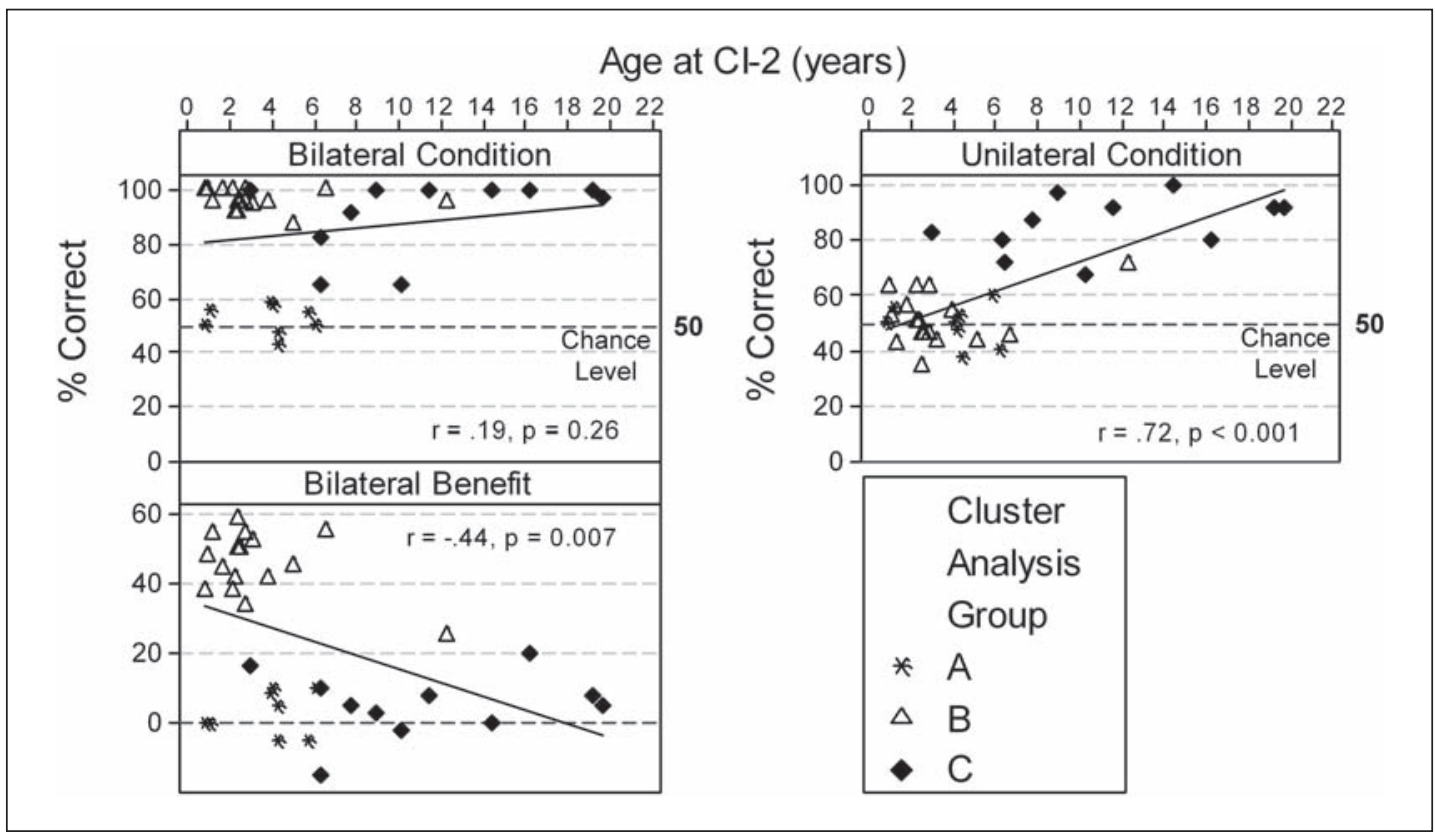

Fig. 3. Percentage of correct responses on the left versus right loudspeaker identification task in the unilateral and bilateral cochlear implant conditions, and the bilateral benefit shown on the task, as a function of age at CI-2 for the 36 participants. Participants are classified as belonging to the Groups A, B and C, which were identified by a cluster analysis as distinct groups within the performance data.

In the unilateral condition these participants were slower and less confident in their responses. In contrast, the participants who scored above chance in both conditions generally performed the task in a much more deliberate way, taking longer and thinking carefully before responding in either device conditions. Many of these participants reported using differences in some aspect of sound quality to identify the presenting loudspeaker in both device conditions.

\section{Discussion}

On the left versus right loudspeaker identification task, only $33.3 \%$ of participants scored above chance in the unilateral condition; the mean score for these 12 participants was $84.7 \%$ (SD: 10.7). In contrast, $77.8 \%$ of participants scored above chance in the bilateral condition; the mean score for these 28 participants was $94.1 \%$ (SD: 9.3). Bilateral benefit was demonstrated by $52.8 \%$ of participants; the mean benefit for these 19 participants was $42.4 \%$ (SD: 11.9). A cluster analysis identified 3 distinct groups within the performance data. Group A $(n=8)$ consisted of participants who performed at chance level in both conditions. Group B $(n=17)$ consisted of participants who performed at chance level, or just above chance level $(\mathrm{n}=1)$, in the unilateral condition but performed significantly better in the bilateral condition, with the majority scoring $>95 \%$. Group C $(n=11)$ consisted of participants who scored significantly above chance level in both conditions; only 2 of these participants demonstrated a bilateral benefit, though ceiling effects may have limited bilateral benefit for an additional 5 participants. Examination of the demographic characteristics of these groups indicated all groups were similar in terms of the age at CI-1 and length of bilateral experience 
(refer to table 3). Group C was older than the other two groups at the time of CI-2 and at testing, and had a longer time between implants, whilst Groups A and B were not significantly different in terms of these factors. Examination of the relationships between these demographic characteristics and performance data across the entire group of 36 participants indicated that the score in the unilateral condition was strongly associated with age at CI-2, age at testing, and time between implants, and was modestly associated with age at CI-1. The score in the bilateral condition was not significantly correlated with any of these factors. Bilateral benefit was negatively, though modestly, associated with age at CI-2, age at testing, and time between implants. It is important to note the high degree of co-linearity amongst these demographic characteristics.

Firstly, considering performance in the unilateral condition, it was not unexpected that the majority of participants performed at chance level. In contrast, performance was above chance for $12(33 \%)$ participants. Eleven of these participants formed Group C, which was older at the time of CI-2 and at testing, and had a longer time between implants (refer to table 3). These characteristics are consistent with the correlation coefficients, which indicated that participants who were older at CI-2 and at testing had a longer time between implants and, to a lesser extent, were older at CI-1, and achieved higher scores in the unilateral condition. As noted, the high degree of co-linearity between these demographic characteristics does not allow the association between any one factor and performance to be clearly established. It is reasonable to assume that being older at CI-1 and/or at CI-2 would not, of itself, result in better performance in the unilateral condition, and that the other demographic characteristics were likely to be more relevant. The participants who were older at testing may have developed the cognitive skills required to identify and remember differences in sound quality and to link these differences to sound presented by one loudspeaker versus the other. This suggestion is consistent with the response behaviour observed and the verbal feedback given during test session; older participants generally made slower, more considered responses in both the unilateral and bilateral conditions, and some reported that there was a sound quality difference which enabled them to identify the presenting loudspeaker. It is also possible that the unilateral performance of this older group in the present study was due to their longer time between implants, which gave them more experience with CI-1 alone, however, this does not explain why they also performed well in the bilateral condition, and it is inconsistent with the finding that superior performance in the unilateral condition was correlated with increased age at CI-1.

In previous lateralisation studies involving only young participants aged less than 6 years, no participant has been reported as performing above chance in the unilateral condition [Beijen et al., 2007; Galvin et al., 2008; Grieco-Calub et al., 2008]. Where older participants have also been included, it has been reported that 41\% [Sparreboom et al., 2011] to 77\% [Litovsky et al., 2006] of participants were able to lateralise in the unilateral condition. In previous studies examining localisation and lateralisation, unilateral performance has not been found to correlate with age at CI-1, time between implants or, in 2 out of 3 studies, age at CI-2 [Grieco-Calub and Litovsky, 2010; Sparreboom et al., 2011; Steffens et al., 2008]; this may have been due to the more limited age range in all of these studies. In the one study which did identify a correlation with age at CI-2, the authors also suggested that the cognitive component of the task resulted in older participants demonstrating superior performance, rather than the age at CI-2 being directly influential [Sparreboom et al., 2011].

Considering the bilateral condition, it was a positive outcome that the majority of participants performed well in this condition. Nevertheless, given that left versus right loudspeaker identification is a relatively simple spatial hearing task, it is of interest that 8 participants (22\%) performed at chance level. These 8 participants formed Group A, which was relatively young at the time of each implant and had a relatively short time between implants, 
including no delay for 2 simultaneously implanted participants (refer to table 3). Group A was not significantly different to Group B, which performed very well in the bilateral condition. This difference in performance, despite similar demographic characteristics, is consistent with the finding of no correlation between performance in the bilateral condition and the demographic characteristics. There is no obvious reason why these 8 participants were unable to lateralise. Across previous studies employing lateralisation tasks, the percentage of participants unable to perform the task in the bilateral condition has varied greatly, ranging from $0 \%$ for a small group of post-meningitic participants with a short duration of deafness [Beijen et al., 2007] through to $60 \%$ for a group of toddlers [Grieco-Calub et al., 2008]. The main points to take from this are that not all children with bilateral implants appear to develop spatial hearing skills, at least in the first 2 years, and that it is difficult to predict outcomes for individuals.

Just over half of the participants demonstrated a significant bilateral benefit. The 2 participants who demonstrated the smallest benefit belonged to Group C, whilst the majority of those demonstrating a bilateral benefit formed Group B. Group B was younger than Group $\mathrm{C}$ at the time of CI-2 and at testing, and had a shorter time between implants. This is consistent with the fact that, for the overall group, bilateral benefit was found to be negatively correlated with age at CI-2, age at testing, and time between implants. The correlations were modest, which is consistent with the fact that not all of the younger children demonstrated a significant benefit, whilst 2 older children in Group C did demonstrate a small, significant benefit. The potential to demonstrate bilateral benefit may be considered to have been limited for older participants, as they generally performed reasonably well in the unilateral condition; however, the correlations with benefit held true even when the 5 older participants with potential ceiling effects were removed from the analysis. Although a more complex spatial hearing task may have been more appropriate for evaluating the abilities of these older participants, the aim of comparing performance across a wide age range necessitated the choice of an assessment task which was also suitable for toddlers.

In only 6 previous studies did the assessment conditions and the reporting of results allow the reader to consider the proportion of participants demonstrating a bilateral benefit. Three studies are comparable with the present result, showing a bilateral benefit for around half to two-thirds of the participants [Galvin et al., 2008; Grieco-Calub and Litovsky, 2010; Litovsky et al., 2006]. The only study to report a much higher rate involved a small group of post-meningitic children with a short duration of deafness [Beijen et al., 2007].

In summary, this study evaluated the ability of implanted toddlers, children and young adults to identify the location of a sound source in a left versus right task when the two loudspeakers were separated by $180^{\circ}$. In the unilateral condition, children who were older at the time of the second implant and/or at testing, and/or had a longer time between implants, achieved higher scores, with $33 \%$ scoring above chance. It was not possible to clearly determine the relationship between individual demographic characteristics and performance due to a high degree of co-linearity amongst the factors, though it was speculated that age at testing may have influenced performance in the unilateral condition. In the bilateral condition, a clear majority of participants scored above chance, but there was no relationship identified with any of the demographic characteristics. When comparing performance in the two conditions, just over half of the participants demonstrated a significant bilateral benefit. Children who were younger at the time of the second implant, and/or at testing, and/or had a shorter time between implants, demonstrated a greater bilateral benefit, although the relationships were modest.

The clinical implications of these results are that children who are younger at the time of their second implant and/or have a shorter time between implants are more likely to gain an improvement in their hearing skills for basic spatial tasks through the use of bilateral im- 
plants. Although the degree to which performance on simple forced-forced choice tasks relates to spatial hearing abilities in real life is difficult to determine, it would be reasonable to predict that children able to perform the test task with more speed and accuracy may also demonstrate superior spatial hearing skills in everyday life, or may have a greater chance of developing superior skills. However, not all young children are able to perform this basic lateralisation task in the first 24 months after switch-on. Some may require more extensive bilateral experience, or it is possible that some may never develop the relevant skills. It is vital that this difficulty in predicting spatial hearing benefits to individuals from bilateral CIs is made clear to families and candidates during pre-operative counselling. Moreover, a small proportion of adolescents will also gain such a benefit from a second implant, and may also demonstrate or develop superior spatial hearing skills in everyday life. Older children, adolescents and young adults have a greater likelihood of being able to perform basic lateralisation tasks using one implant alone in the testing situation. The extent to which this ability is beneficial in everyday life, where target stimuli are far more varied, is very unclear. In the future, as children who were bilaterally implanted at a young age become capable of more complex testing, it will be important to assess their spatial hearing skills using more complex tasks. This will improve our understanding of the potential benefits of bilateral implants, and of who is most likely to gain those benefits. Given the variability in performance across individuals, and the fact that reported associations between performance and demographic characteristics have varied across studies, larger sample sizes than those involved to date are needed. In addition, longitudinal data, including the results of assessments conducted soon after bilateral implantation, will provide information regarding the development of spatial hearing skills.

\section{Acknowledgements}

The authors are very grateful to the children and families who participated in this research, and to the staff of the Royal Victorian Eye and Ear Hospital, Cochlear Implant Clinic. Thanks are also due to Dr. Richard Van Hoesel for providing the software and helpful comments, and to Mark Harrison for technical support. Ethical approval for this work was given by the Human Research Ethics Committee of the Royal Victorian Eye and Ear Hospital, Melbourne (project No. 02/506H/07).

\section{Disclosure Statement}

Financial support for this work was provided by the National Health and Medical Research Council (project grant No. 454318); Audiology, Hearing and Speech Science, Department of Otolaryngology, University of Melbourne; the Bionic Ear Institute, Melbourne; the Royal Victorian Eye and Ear Hospital, Melbourne; the William Angliss Foundation; and the Collier Fund. The authors have no conflicts of interest to declare.

\section{References}

Beijen JW, Mylanus EAM, Snik AFM: Sound localization ability of young children with bilateral cochlear implants. Otol Neurotol 2007;28:479-485.

Galvin KL, Mok M, Dowell RC: Perceptual benefit and functional outcomes for children using sequential bilateral cochlear implants. Ear Hear 2007a;28:470-482. 
Galvin KL, Mok M, Dowell RC, Briggs RJ: 12-month post-operative results for older children using sequential bilateral implants. Ear Hear 2007b;28:19S-21S.

Galvin KL, Mok M, Dowell RC, Briggs RJ: Speech detection and localization results and clinical outcomes for children receiving sequential bilateral cochlear implants before four years of age. Int J Audiol 2008;47:636-646.

Grieco-Calub TM, Litovsky RY: Sound localization skills in children who use bilateral cochlear implants and in children with normal acoustic hearing. Ear Hear 2010;31:645-656.

Grieco-Calub TM, Litovsky RY, Werner LA: Using the observer-based psychophysical procedure to assess localization acuity in toddlers who use bilateral cochlear implants. Otol Neurotol 2008;29:235-239.

Laske RD: Subjective and objective results after bilateral cochlear implantation in adults. Am J Otol 2009; 30:313.

Litovsky RY, Johnstone PM, Godar S, Agrawal S, Parkinson AJ, Peters R, Lake J: Bilateral cochlear implants in children: localization acuity measured with minimum audible angle. Ear Hear 2006;27: 43-59.

Lovett RE, Kitterick PT, Hewitt CE, Summerfield AQ: Bilateral or unilateral cochlear implantation for deaf children: an observational study. Arch Dis Child 2010;95:107-112.

Sparreboom M, Snik AFM, Mylanus EAM: Sequential bilateral cochlear implantation in children: development of the primary auditory abilities of bilateral stimulation. Audiol Neurotol 2011;16:203-213.

Steffens T, Lesinski-Schiedat A, Strutz J, Aschendorff A, Klenzner T, Ruhl S, Voss B, Wesarg T, Laszig R, Lenarz T: The benefits of sequential bilateral cochlear implantation for hearing-impaired children. Acta Oto-Laryngologica 2008;128:164-176.

Van Deun L, van Wieringen A, Scherf F, Deggouj N, Desloovere C, Offeciers FE, Van de Heyning PH, Dhooge IJ, Wouters J: Earlier intervention leads to better sound localization in children with bilateral cochlear implants. Audiol Neurotol 2010;15:7-17.

van Hoesel R, Tyler RS: Speech perception, localization and lateralization with bilateral cochlear implants. J Acoust Soc Am 2003;113:1617-1630.

Verschuur C, Lutman M, Ramsden R, Greenham P, O’Driscoll M: Auditory localization abilities in bilateral cochlear implant recipients. Otol Neurotol 2005;26:965. 